\title{
Recent CMS B physics results
}

\author{
Sergey Polikarpov on behalf of the CMS Collaboration ${ }^{1, a}$ \\ ${ }^{1}$ National Research Nuclear University "Moscow Engineering Physics Institute” (MEPhl), Moscow, Russia
}

\begin{abstract}
Various recent CMS B physics results are reported, including measurement of B hadron lifetimes; observation of $\Upsilon(1 \mathrm{~S})$ pair production; angular analysis of the rare decay $\mathrm{B}^{0} \rightarrow \mathrm{K}^{* 0} \mu^{+} \mu^{-}$; search for $\mathrm{X}(5568)$ state. All analyses are performed using data collected by the CMS experiment at the LHC in pp collisions at $\sqrt{s}=8 \mathrm{TeV}$, which corresponds to an integrated luminosity of about $20 \mathrm{fb}^{-1}$.
\end{abstract}

\section{Introduction}

The CMS experiment [1] continues contributing to the Heavy Flavour physics sector. In this report, several recent CMS results on B hadron and quarkonium production and properties are discussed, including:

- Precise measurements of $\mathrm{B}^{0}, \mathrm{~B}_{\mathrm{s}}^{0}, \mathrm{~B}_{\mathrm{c}}^{+}$and $\Lambda_{\mathrm{b}}^{0}$ lifetimes in the decay channels with $\mathrm{J} / \psi \rightarrow \mu^{+} \mu^{-}$, in particular: $\mathrm{B}^{0} \rightarrow \mathrm{J} / \psi \mathrm{K}^{* 0}\left(\mathrm{~K}^{* 0} \rightarrow \mathrm{K}^{+} \pi^{-}\right), \mathrm{B}^{0} \rightarrow \mathrm{J} / \psi \mathrm{K}_{S}^{0}\left(\mathrm{~K}_{S}^{0} \rightarrow \pi^{+} \pi^{-}\right), \mathrm{B}_{\mathrm{s}}^{0} \rightarrow \mathrm{J} / \psi \phi\left(\phi \rightarrow \mathrm{K}^{+} \mathrm{K}^{-}\right)$, $\mathrm{B}_{\mathrm{s}}^{0} \rightarrow \mathrm{J} / \psi \pi^{+} \pi^{-}, \mathrm{B}_{\mathrm{c}}^{+} \rightarrow \mathrm{J} / \psi \pi^{+}$and $\Lambda_{\mathrm{b}}^{0} \rightarrow \mathrm{J} / \psi \Lambda^{0}\left(\Lambda^{0} \rightarrow \mathrm{p} \pi^{-}\right)$.

- First observation of $\Upsilon(1 \mathrm{~S})$ pair production in proton-proton collisions and measurement of its cross section.

- Measurement of the $\mathrm{P}_{1}$ and $\mathrm{P}_{5}^{\prime}$ angular parameters of the decay $\mathrm{B}^{0} \rightarrow \mathrm{K}^{* 0} \mu^{+} \mu^{-}$.

- Search for $\mathrm{X}(5568)$ state decaying into $\mathrm{B}_{\mathrm{s}}^{0} \pi^{ \pm}$.

\section{Precise measurements of $B$ hadron lifetimes}

Models inspired by Quantum Chromodynamics (QCD) provide precise predictions for b-hadron lifetimes. Accurate experimental measurements are essential to test those predictions.

$\mathrm{B}$ hadron decays are reconstructed using the decay $\mathrm{J} / \psi \rightarrow \mu^{+} \mu^{-}$, which also triggers the event readout. The $\mathrm{J} / \psi$ candidate is combined with a track (for $\mathrm{B}_{\mathrm{c}}^{+} \rightarrow \mathrm{J} / \psi \pi^{+}$case) or with two tracks of opposite charge (for $\mathrm{B}^{0} \rightarrow \mathrm{J} / \psi \mathrm{K}^{* 0}, \mathrm{~B}_{\mathrm{s}}^{0} \rightarrow \mathrm{J} / \psi \pi^{+} \pi^{-}$and $\mathrm{B}_{\mathrm{s}}^{0} \rightarrow \mathrm{J} / \psi \phi$ ) to form a vertex, which is required to be significantly displaced from the primary vertex (PV). For $\Lambda_{\mathrm{b}}^{0} \rightarrow \mathrm{J} / \psi \Lambda^{0}$ and $\mathrm{B}^{0} \rightarrow \mathrm{J} / \psi \mathrm{K}_{S}^{0}$ decays, the long-lived hadrons are reconstructed from two-prong displaced vertices (in case of $\Lambda^{0} \rightarrow \mathrm{p} \pi^{-}$, the track with higher momentum of the two is selected as a proton candidate). Thresholds on the track transverse momentum, track and vertex fits quality and masses of the intermediate states are applied to reduce the background.

ae-mail: sergey.polikarpov@cern.ch 


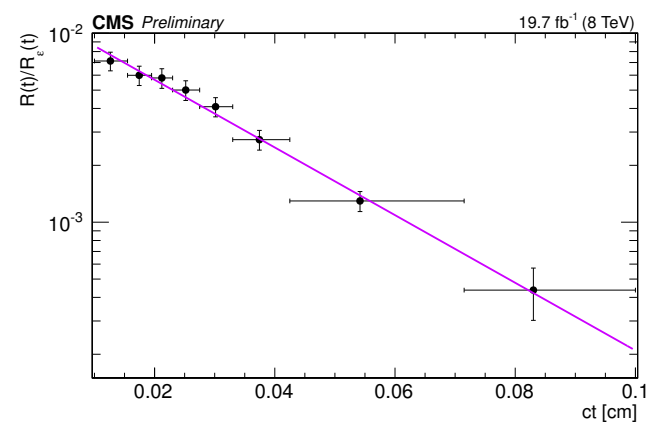

Figure 1. Ratio of efficiency-corrected $c t$ distributions for $\mathrm{B}_{\mathrm{c}}^{+}$and $\mathrm{B}^{+}$signals [2]. The line shows the fit result with an exponential function.

The lifetimes are extracted with a fit to the 3-dimensional distribution of the reconstructed invariant mass of B hadron candidate, $c t$ and per-event $c t$ uncertainty. Here $t$ is the measured proper decay time of a B hadron candidate, calculated using the expression

$$
t=L_{x y} \frac{M}{p_{\mathrm{T}}}
$$

where $L_{x y}\left(p_{\mathrm{T}}\right)$ is the candidate transverse decay length (transverse momentum) and $M$ is the worldaverage mass of $\mathrm{B}$ hadron. The fit function includes the signal component, the background component and, in case of the $\mathrm{B}_{\mathrm{s}}^{0} \rightarrow \mathrm{J} / \psi \pi^{+} \pi^{-}$channel, the contribution from other partially reconstructed or misidentified $\mathrm{B}$ hadron decays. The reconstruction efficiency dependence on $c t$ is taken into account in the fits.

For the $\mathrm{B}_{\mathrm{c}}^{+}$lifetime, a different approach is used to reduce systematic uncertainties. The yields of $\mathrm{B}_{\mathrm{c}}^{+} \rightarrow \mathrm{J} / \psi \pi^{+}$and $\mathrm{B}^{+} \rightarrow \mathrm{J} / \psi \mathrm{K}^{+}$decays are obtained in intervals of $c t$ and their ratio is fit with an exponential function, as shown in Fig. 1. The $\mathrm{B}_{\mathrm{c}}^{+}$lifetime is obtained using the known lifetime of the $\mathrm{B}^{+}$meson and the fit result. The same method was used by $\mathrm{LHCb}$ collaboration to measure the $\mathrm{B}_{\mathrm{c}}^{+}$ lifetime in the same decay mode [3].

Dominant systematic uncertainties come from the finite size of the simulation sample, algorithm of PV selection and possible detector misalignment.

The measured lifetimes are [2]:

$$
\begin{aligned}
& \left.c \tau_{\mathrm{B}^{0}}=453.0 \pm 1.6 \text { (stat) } \pm 1.5 \text { (syst) } \mu \mathrm{m} \text { (in } \mathrm{J} / \psi \mathrm{K}^{* 0}\right), \\
& c \tau_{\mathrm{B}^{0}}=457.8 \pm 2.7 \text { (stat) } \pm 2.7 \text { (syst) } \mu \mathrm{m} \text { (in } \mathrm{J} / \psi \mathrm{K}_{S}^{0} \text { ), } \\
& c \tau_{\mathrm{B}_{\mathrm{s}}^{0}}=502.7 \pm 10.2 \text { (stat) } \pm 3.2 \text { (syst) } \mu \mathrm{m} \text { (in } \mathrm{J} / \psi \pi^{+} \pi^{-} \text {), } \\
& c \tau_{\mathrm{B}_{\mathrm{s}}^{0}}=443.9 \pm 2.0 \text { (stat) } \pm 1.2 \text { (syst) } \mu \mathrm{m} \text { (in } \mathrm{J} / \psi \phi \text { ), } \\
& c \tau_{\Lambda_{\mathrm{b}}^{0}}=442.9 \pm 8.2 \text { (stat) } \pm 2.7 \text { (syst) } \mu \mathrm{m} \text {, } \\
& c \tau_{\mathrm{B}_{\mathrm{c}}^{+}}=162.3 \pm 8.2 \text { (stat) } \pm 4.7 \text { (syst) } \pm 0.1\left(\tau_{\mathrm{B}^{+}}\right) \mu \mathrm{m} \text {, }
\end{aligned}
$$

where the first (second) uncertainty is statistical (systematic). Each result is consistent with the current world average value and the accuracy in each channel is at the level of or better than the previous results. 

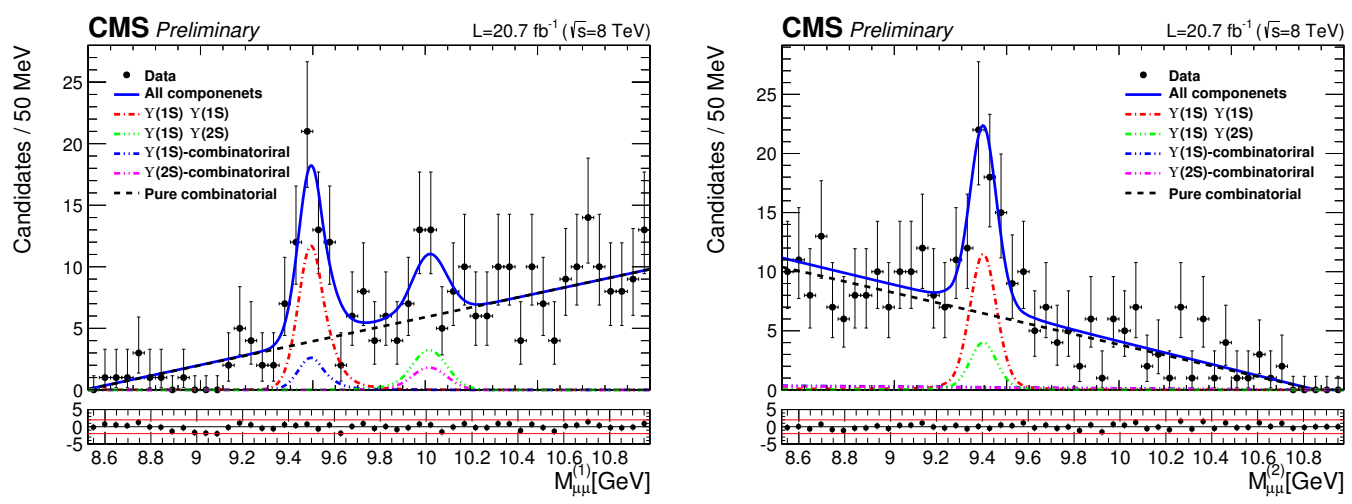

Figure 2. Projections of the 2D fit. Left: invariant mass distribution of heavier dimuon $\left(M_{\mu^{+} \mu^{-}}^{1}\right)$ and right: invariant mass distribution of lighter dimuon $M_{\mu^{+} \mu^{-}}^{1}$ [4].

\section{Observation of $\Upsilon(1 S)$ pair production}

Quarkonium pair production cross section measurements are one of the key measurements in understanding Single Parton Scattering (SPS) and Double Parton Scattering (DPS) contributions. The observation of simultaneous production of two $\Upsilon(1 \mathrm{~S})$ mesons in pp collisions at $\sqrt{s}=8 \mathrm{TeV}$ is reported here. The dimuon decay is used to reconstruct $\Upsilon$ candidates. Four muons are required to have a common vertex, and the rapidity of the reconstructed $\Upsilon$ candidates is required to be $|y(\Upsilon)|<2.0$.

A two-dimensional unbinned fit to the invariant mass of reconstructed $M_{\mu^{+} \mu^{-}}^{1}$ and $M_{\mu^{+} \mu^{-}}^{2}$ combinations is used to extract the signal yield (where $M_{\mu^{+} \mu^{-}}^{1}>M_{\mu^{+} \mu^{-}}^{2}$ ). Events containing more than one $\Upsilon \Upsilon$ candidates are discarded. No signs of $\Upsilon(3 S)$ and higher states are observed, therefore, their contributions are neglected. The fit includes pure combinatorial background component, $\Upsilon(1 \mathrm{~S}) \Upsilon(1 \mathrm{~S})$ and $\Upsilon(2 S) \Upsilon(1 S)$ signal components and the contributions from $\Upsilon(1 S)$ or $\Upsilon(2 S)$ and two unassociated muons. The fit result is presented in Fig. 2 and returns the number of $\Upsilon(1 \mathrm{~S}) \Upsilon(1 \mathrm{~S})$ signal candidates $N_{s i g 1}=38 \pm 7$ with a corresponding significance of $9.6 \sigma$ (including systematic uncertainties) [4].

The cross section $\sigma_{\mathrm{T}}$ of $\Upsilon(1 \mathrm{~S})$ pair production is found to be $\sigma_{\mathrm{T}}=68.8 \pm 12.7$ (stat) \pm 7.4 (syst) \pm $2.8(\mathrm{BR}) \mathrm{pb}[4]$ in the region $|y(\Upsilon)|<2.0, p_{\mathrm{T}}(\Upsilon(1 \mathrm{~S}))<50 \mathrm{GeV}$, assuming an isotropic decay of the $\Upsilon(1 \mathrm{~S})$, where the uncertainties are statistical, systematic and from the branching fraction knowledge, respectively. Cross section ranges from $-38 \%$ to $+36 \%$ from the value above when different $\Upsilon(1 \mathrm{~S})$ polarizations are assumed.

\section{Measurement of $\mathrm{P}_{1}$ and $\mathrm{P}_{5}^{\prime}$ angular parameters in the decay $\mathrm{B}^{0} \rightarrow \mathrm{K}^{* 0} \mu^{+} \mu^{-}$}

Rare decays that proceed via flavor-changing neutral current (FCNC) provide a sensitive laboratory to study the effects of New Physics (NP), since these decays are suppressed in the Standard Model (SM). In particular, NP can modify the angular distributions of the $\mathrm{B}^{0} \rightarrow \mathrm{K}^{* 0} \mu^{+} \mu^{-}$decay. Some tensions w.r.t. SM was observed in $\mathrm{P}_{5}^{\prime}$ parameter by Belle [5] and $\mathrm{LHCb}$ [6].

CMS performs an angular analysis of this decay using a data set of approximately $20.7 \mathrm{fb}^{-1} \mathrm{pp}$ collisions collected at $\sqrt{s}=8 \mathrm{TeV}$. A dedicated trigger that searches for dimuon vertices not consistent with the pp collision region is used, and events from $\mathrm{B}^{0} \rightarrow \mathrm{J} / \psi(\psi(2 \mathrm{~S})) \mathrm{K}^{* 0}$ decays are used as control samples. 

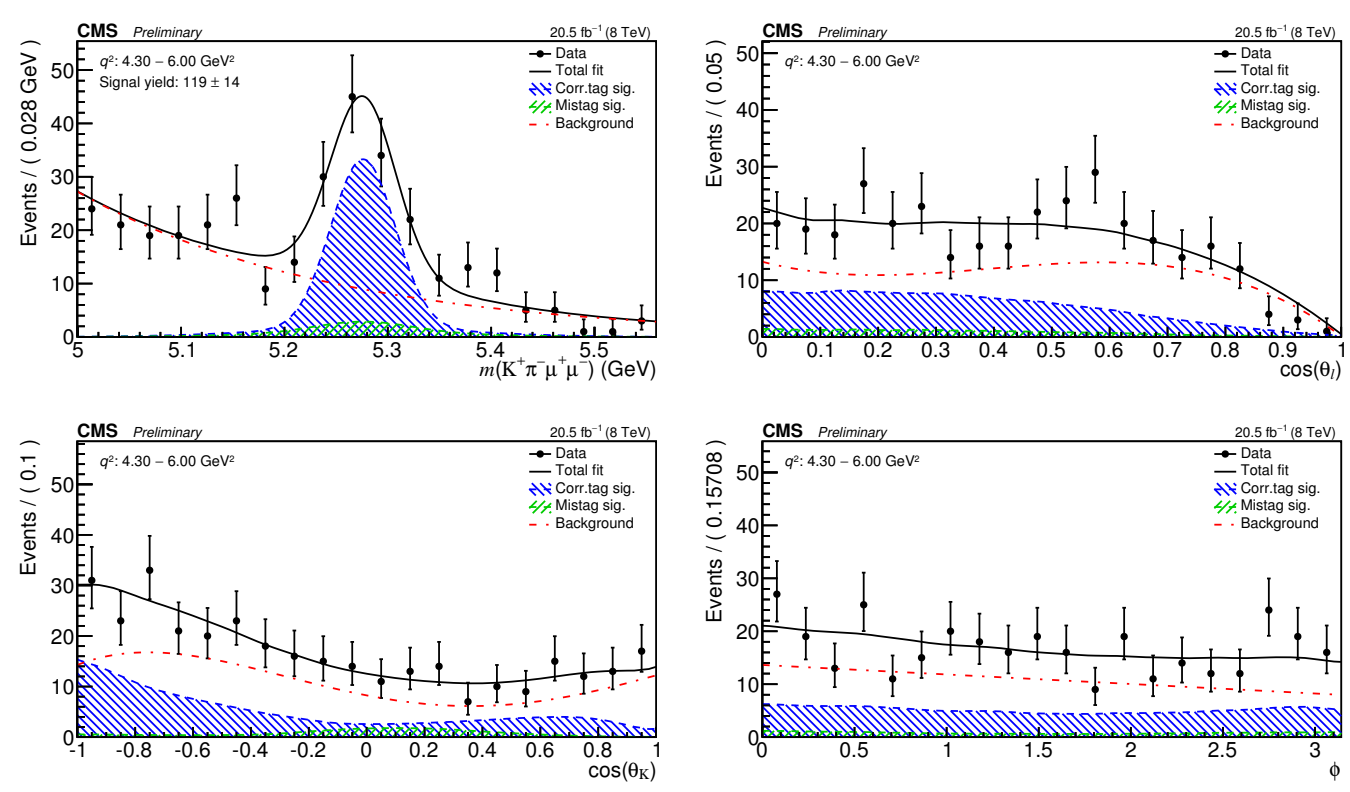

Figure 3. Distributions of $\mathrm{K}^{+} \pi^{-} \mu^{+} \mu^{-}$mass and three angular variables in the third bin of $q^{2}\left(4.30<q^{2}<\right.$ $6.00 \mathrm{GeV}^{2}$ ) from the decay $\mathrm{B}^{0} \rightarrow \mathrm{K}^{* 0} \mu^{+} \mu^{-}[7]$.

The B hadron reconstruction is similar to the procedure described in Sect. 2. Kaon and pion masses are assigned to the two tracks so that the combination with invariant mass closer to the known $\mathrm{K}^{* 0}$ mass is retained (mistag probability of this procedure is about 14\%). After all selection requirements, 1397 events are remaining in the signal sample (contributions from $\mathrm{J} / \psi \rightarrow \mu^{+} \mu^{-}$and $\psi(2 \mathrm{~S}) \rightarrow \mu^{+} \mu^{-}$ are removed).

The $\mathrm{P}_{1}$ and $\mathrm{P}_{5}^{\prime}$ observables are studies in 7 bins of the square of dimuon invariant mass, $q^{2}$. In each $q^{2}$ bin an unbinned extended maximum-likelihood fit is performed to four variables: $\mathrm{K}^{+} \pi^{-} \mu^{+} \mu^{-}$ invariant mass, $\theta_{l}$ (the angle between $\mu^{+}$momentum and the direction opposite to the $\mathrm{B}^{0}$ in the dimuon rest frame), $\theta_{K}$ (the angle between $\mathrm{K}^{+}$momentum and the direction opposite to the $\mathrm{B}^{0}$ in the $\mathrm{K}^{+} \pi^{-}$ rest frame), and $\phi$ (the angle between the plane containing the two muons and the plane containing the kaon and pion in the $\mathrm{B}^{0}$ rest frame). The fit function consists of correctly tagged signal component, mistagged signal component and background component. The signal function includes $\mathrm{S}$-wave $\mathrm{K}^{+} \pi^{-}$ component, $\mathrm{P}$-wave $\mathrm{K}^{* 0} \rightarrow \mathrm{K}^{+} \pi^{-}$component and their interference. The efficiency dependence on the angular parameters is accounted in the fits, and the background shape is obtained from the $\mathrm{B}^{0}$ sideband. The fit function depends on 6 parameters of angular distributions: $F_{L}, F_{S}, A_{S}, A_{S}^{5}, \mathrm{P}_{1}$ and $\mathrm{P}_{5}^{\prime}$, where the first three of them $\left(F_{L}, F_{S}\right.$ and $\left.A_{S}\right)$ are fixed to the values measured in Ref. [8] to ensure the fit convergence given the limited number of candidates. The result of the fit in the third $q^{2}$ bin $\left(4.30<q^{2}<6.00 \mathrm{GeV}^{2}\right)$ is presented in Fig. 3 .

The dominant sources of systematic uncertainties are the fit bias (evaluated with fits to the simulated data), the uncertainties on $F_{L}, F_{S}$ and $A_{S}$, the finite size of the simulated data sample, and the $\mathrm{K}^{+} \pi^{-}$mistagging (estimated using control channel $\mathrm{B}^{0} \rightarrow \mathrm{J} / \psi \mathrm{K}^{* 0}$ ). 

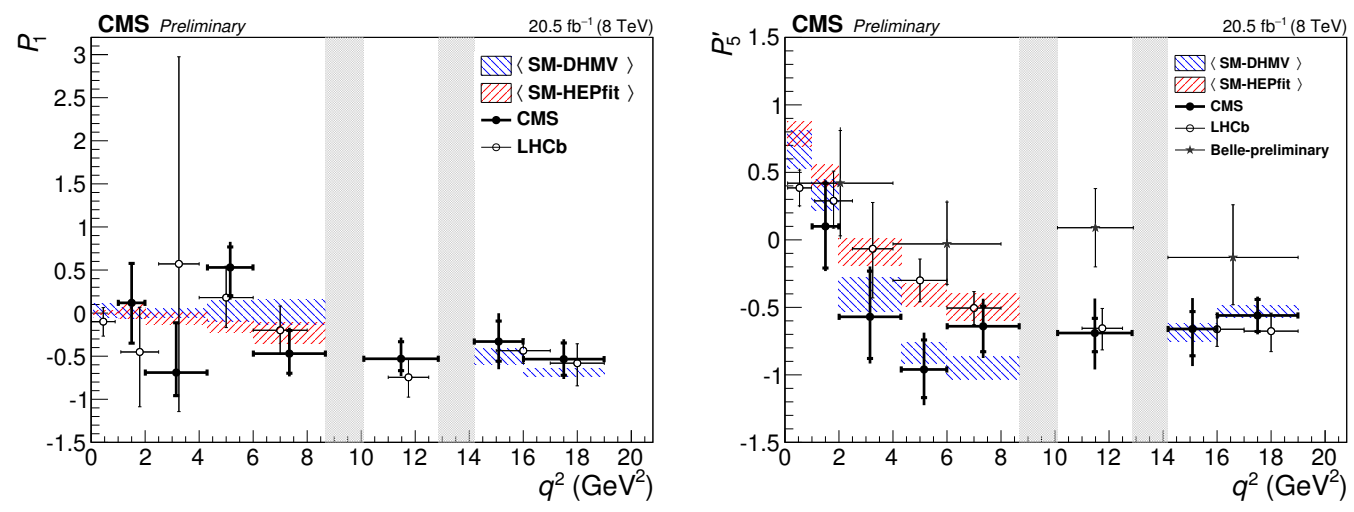

Figure 4. Measured values of $\mathrm{P}_{1}$ and $\mathrm{P}_{5}^{\prime}$ as a function of $q^{2}$ from the $\mathrm{B}^{0} \rightarrow \mathrm{K}^{* 0} \mu^{+} \mu^{-}$decay [7], compared with $\mathrm{LHCb}[6]$ and Belle [5] results. The vertical shaded regions correspond to the $\mathrm{J} / \psi$ and $\psi(2 \mathrm{~S})$ regions.

The resulting values of $\mathrm{P}_{1}$ and $\mathrm{P}_{5}^{\prime}$ in bins of $q^{2}$ are presented in Fig. 4 along with the results from Belle [5] and LHCb [6]. The SM theoretical predictions available for comparison are predictions from SM-DHMV and SM-HEPfit. The CMS measurements are in agreement with both theoretical predictions.

\section{Search for $X(5568)$}

The claim for the observation of the $\mathrm{X}(5568)$ state decaying into $\mathrm{B}_{\mathrm{s}}^{0} \pi^{ \pm}$by the D0 Collaboration [9] raised many controversial discussions in the exotic hadron community. The new state was reported to have a mass and natural width of $5567.8 \pm 2.9$ (stat $)_{-1.9}^{+0.9}$ (syst) $\mathrm{MeV}$ and $21.9 \pm 6.4(\text { stat })_{-2.5}^{+5.0}$ (syst) $\mathrm{MeV}$, respectively. The relative production of $\mathrm{X}(5568)$ w.r.t. $\mathrm{B}_{\mathrm{s}}^{0}$ meson, multiplied by $\mathcal{B}\left(\mathrm{X}(5568) \rightarrow \mathrm{B}_{\mathrm{s}}^{0} \pi^{ \pm}\right)$, was measured to be $\rho_{\mathrm{X}}=(8.6 \pm 1.9 \pm 1.4) \%$ for $p_{\mathrm{T}}\left(\mathrm{B}_{\mathrm{s}}^{0}\right)>10 \mathrm{GeV}$ in proton-antiproton collisions at $\sqrt{s}=1.96 \mathrm{TeV}$ [9]. The LHCb Collaboration did not find any evidence for $\mathrm{X}(5568)$ in pp collisions at $\sqrt{s}=8 \mathrm{TeV}$ and set an upper limit on $\rho_{\mathrm{X}}: \rho_{\mathrm{X}}<2.4 \% @ 95 \% \mathrm{CL}$ for $p_{\mathrm{T}}\left(\mathrm{B}_{\mathrm{s}}^{0}\right)>10 \mathrm{GeV}$ [10].

We report a negative search result for $\mathrm{X}(5568)$ at the CMS experiment. The $\mathrm{B}_{\mathrm{s}}^{0}$ candidates are reconstructed using the decay channel $\mathrm{B}_{\mathrm{s}}^{0} \rightarrow \mathrm{J} / \psi \phi$, where $\mathrm{J} / \psi \rightarrow \mu^{+} \mu^{-}$and $\phi \rightarrow \mathrm{K}^{+} \mathrm{K}^{-}$decays are used. The same trigger used in the B hadrons lifetime measurement (Sect. 2) is used in this analysis.

About $50,000 \mathrm{~B}_{\mathrm{s}}^{0} \rightarrow \mathrm{J} / \psi \phi$ signal candidates [11] are found in the reconstructed $\mathrm{J} / \psi \phi$ invariant mass distribution. The pions coming from the $\mathrm{B}_{\mathrm{s}}^{0}$ production $\mathrm{PV}$ and satisfying $p_{\mathrm{T}}>0.5 \mathrm{GeV}$ requirement are combined with $\mathrm{B}_{\mathrm{s}}^{0}$ candidates to form $\mathrm{B}_{\mathrm{s}}^{0} \pi^{ \pm}$candidates. The $\mathrm{B}_{\mathrm{s}}^{0} \pi^{ \pm}$invariant mass distribution is shown in Fig. 5 (a), compared between the $\mathrm{B}_{\mathrm{s}}^{0}$ signal region and the $\mathrm{B}_{\mathrm{s}}^{0}$ sideband regions. No significant structures are visible near $5568 \mathrm{MeV}$ nor in the whole mass region $5.5-5.9 \mathrm{GeV}$. The distribution from the $\mathrm{B}_{\mathrm{s}}^{0}$ signal region is close to that of $\mathrm{B}_{\mathrm{s}}^{0}$ sidebands. The fit to the distribution in the $\mathrm{B}_{\mathrm{s}}^{0}$ signal region with a smooth background function and a signal component (Relativistic BreitWigner with mass and width fixed to the values measured by the D0, convolved with resolution) is presented in Fig. 5 (b) and returns a yield of X(5568) signal candidates consistent with zero: $N_{\mathrm{X}}=$ $-175 \pm 134$ [11]. Some variations of selection criteria are made, in particular, different requirements on $\mathrm{B}_{\mathrm{s}}^{0}, \pi^{ \pm}, \mathrm{B}_{\mathrm{s}}^{0} \pi^{ \pm}$candidates $p_{\mathrm{T}}$ or reconstruction quality criteria are imposed. In every case the fit returns the signal yield consistent with zero. The reconstruction procedure is verified by removing the 

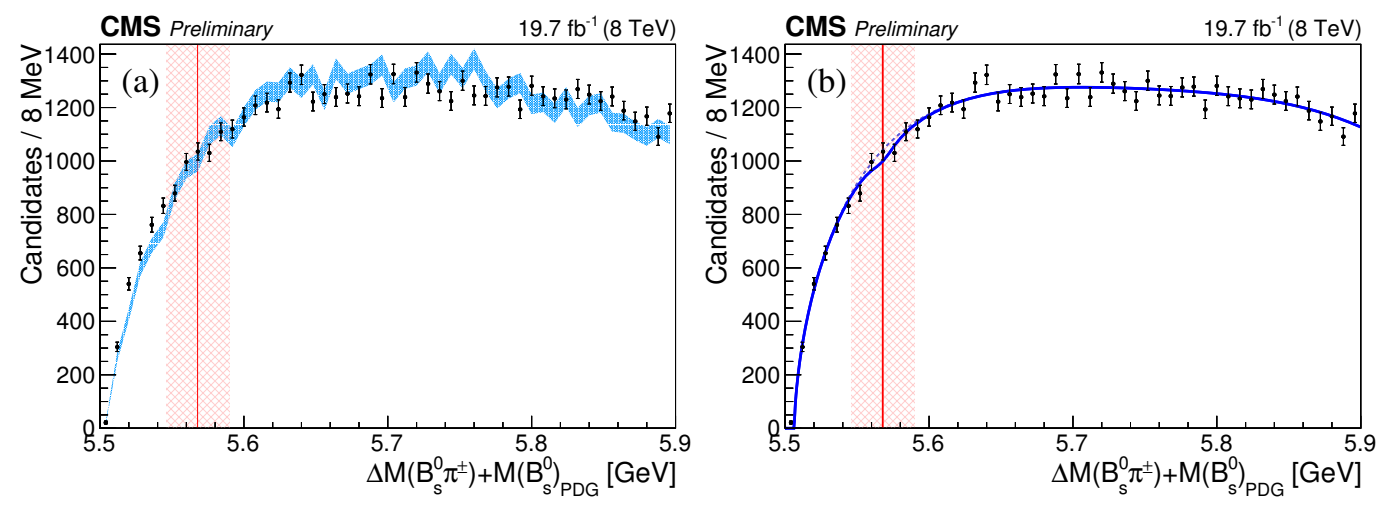

Figure 5. (a): $\mathrm{B}_{\mathrm{s}}^{0} \pi^{ \pm}$invariant mass distribution for the $\mathrm{B}_{\mathrm{s}}^{0}$ signal region and $\mathrm{B}_{\mathrm{s}}^{0}$ sidebands. (b): fit to the $\mathrm{B}_{\mathrm{s}}^{0} \pi^{ \pm}$ invariant mass distribution in the $\mathrm{B}_{\mathrm{s}}^{0}$ signal region [11].

requirements on $\mathrm{K}^{+} \mathrm{K}^{-}$mass, which allows the $\mathrm{B}^{0} \rightarrow \mathrm{J} / \psi \mathrm{K}^{+} \pi^{-}$decay to contribute to the reconstructed $\mathrm{B}_{\mathrm{s}}^{0} \rightarrow \mathrm{J} / \psi \mathrm{K}^{+} \mathrm{K}^{-}$sample. Consequently, the decays of excited $\mathrm{B}$ meson states $\mathrm{B}_{1,2}^{(*)+} \rightarrow \mathrm{B}^{(*) 0} \pi^{+}$produce clear excess in the reconstructed $\mathrm{B}_{\mathrm{s}}^{0} \pi^{ \pm}$invariant mass distribution [11].

An upper limit on $\rho_{\mathrm{X}}$ is obtained: $\rho_{\mathrm{X}}<3.9 \% @ 95 \% \mathrm{CL}$ for $p_{\mathrm{T}}\left(\mathrm{B}_{\mathrm{s}}^{0}\right)>10 \mathrm{GeV}$ [11]. The main source of systematic uncertainty is the knowledge of the efficiency ratio $\epsilon_{\mathrm{X}} / \epsilon_{\mathrm{B}_{\mathrm{s}}^{0}}$ from the simulation.

\section{Summary and acknowledgments}

In summary, CMS presents recent Heavy Flavour physics results. The measured lifetimes of B hadrons are among the most precise to date. First observation of $\Upsilon(1 \mathrm{~S})$ pair production in proton proton collisions is made and the corresponding cross section is measured. The $\mathrm{P}_{1}$ and $\mathrm{P}_{5}^{\prime}$ angular parameters of the rare decay $\mathrm{B}^{0} \rightarrow \mathrm{K}^{* 0} \mu^{+} \mu^{-}$are measured with precision at the level of the best previous measurements, and they are found to be in agreement with the Standard Model predictions and previous results from $\mathrm{LHCb}$ and Belle collaborations, thus no evidence for physics beyond the SM is obtained. The search for $\mathrm{X}(5568)$ is presented, and an upper limit is set on the relative production in agreement with the LHCb collaboration.

The author's work was supported by the Russian Ministry of Education and Science contracts 3.2989.2017 and 14.A12.31.0006. The author would like to thank the CMS Collaboration for the content of the presentation and the ICNFP 2017 conference organizers for opportunity to present these results and warm hospitality.

\section{References}

[1] The CMS Collaboration, JINST 3, S08004 (2008).

[2] The CMS Collaboration, CMS-PAS-BPH-13-008, arXiv:1710.08949 (Submitted to Eur. Phys. J. C.).

[3] LHCb Collaboration, Phys. Lett. B 742, 29-37 (2015).

[4] The CMS Collaboration, JHEP 05, 013 (2017).

[5] LHCb Collaboration, Phys.Rev.Lett.118 no. 11, 111801 (2017). 
[6] LHCb Collaboration, JHEP02, 104 (2016).

[7] The CMS Collaboration, CMS-PAS-BPH-15-008, arXiv:1710.02846 (Submitted to Phys. Lett. B).

[8] The CMS Collaboration, Phys. Lett. B753, 424 (2016).

[9] D0 Collaboration, Phys. Rev. Lett. 117, 022003 (2016).

[10] LHCb Collaboration, Phys. Rev. Lett. 117, 152003 (2016).

[11] The CMS Collaboration, CMS-PAS-BPH-16-002. 